\title{
Magnetogama: an open schematic magnetometer
}

\author{
Wahyudi $^{1}$, Nurul Khakhim ${ }^{2}$, Tri Kuntoro ${ }^{3}$, Djati Mardiatno ${ }^{2}$, Afif Rakhman ${ }^{1}$, Anas Setyo Handaru ${ }^{1}$, \\ Adien Akhmad Mufaqih ${ }^{1}$, and Theodosius Marwan Irnaka ${ }^{1}$ \\ ${ }^{1}$ Geophysics Laboratory, Universitas Gadjah Mada, Yogyakarta, Indonesia \\ ${ }^{2}$ Faculty of Geography, Universitas Gadjah Mada, Yogyakarta, Indonesia \\ ${ }^{3}$ Electronics and Instrumentation Laboratory, Universitas Gadjah Mada, Yogyakarta, Indonesia
}

Correspondence to: Wahyudi (wahyudi_mipa@ugm.ac.id)

Received: 1 November 2016 - Discussion started: 6 February 2017

Revised: 13 July 2017 - Accepted: 26 July 2017 - Published: 5 September 2017

\begin{abstract}
Magnetogama is an open schematic handassembled fluxgate magnetometer. Compared to another magnetometer, Magnetogama has more benefit concerning its price and its ease of use. Practically Magnetogama can be utilized either in land or attached to an unmanned aerial vehicle (UAV). Magnetogama was designed to give open access to a cheap and accurate alternative to magnetometer sensor. Therefore it can be used as a standard design which is directly applicable to the low-budget company or education purposes. Schematic, code and several verification tests were presented in this article ensuring its reproducibility. Magnetogama has been tested with two kind of tests: a comparison with two nearest observatories at Learmonth (LRM) and Kakadu (KDU) and the response of magnetic substance.
\end{abstract}

\section{Introduction}

A magnetometer is a device which can record magnetic field at the specific location where the measurement is conducted. Several types of magnetometer exist, such as proton precession magnetometer (PPM) (Serson, 1962; Tyagi et al., 1983), fluxgate magnetometer (Primdahl, 1979), superconducting quantum interference device (SQUID) (Drung et al., 1990; Fagaly, 2006; Cleuziou et al., 2006) and Hall magnetometer (Peeters and Li, 1998). The fluxgate magnetometer has advantages over PPM in terms of measuring magnetic field amplitude and direction, and it has a higher sampling rate. With the main purpose of measuring the earth's magnetic field, the fluxgate magnetometer has sufficient accuracy in a compact form; the SQUID magnetometer, in contrast, needs liquid helium to operate but has very high accuracy.
Geophysicists need a magnetometer which is portable and has sufficient accuracy. The PPM and fluxgate are two types of magnetometers that meet these criteria. A commercial magnetometer is usually already equipped with a data logger and acquisition system. Those magnetometers have been calibrated, tested and are ready to be used in exploration with sufficient accuracy and stability. Unfortunately, with all those benefits, the price is usually quite high. This aspect is disadvantageous for those beginning to learn geomagnetic methods.

Magnetogama is a device which combines a proprietary fluxgate magnetic sensor with a widely available microcontroller and an analog-to-digital converter (ADC). It has an open schematic, algorithm and source code, enabling everyone to build their version of a magnetometer. Magnetogama is easy to assemble and beneficial compared to the other complete magnetometers concerning price and suitability for both general and unmanned aerial vehicle (UAV) magnetic surveys. Magnetogama is designed to be as efficient and effective as possible. Besides the advantage of accurate reading, Magnetogama also has an easy-to-use interface. Magnetic reading and location data can be stored automatically in its nonvolatile memory.

\section{Components}

In order to properly function as a complete magnetometer, there are at least six main components that must be assembled, i.e., magnetic sensor, microcontroller, ADC, data storage, positioning device, power supply and user input-output (I/O). 


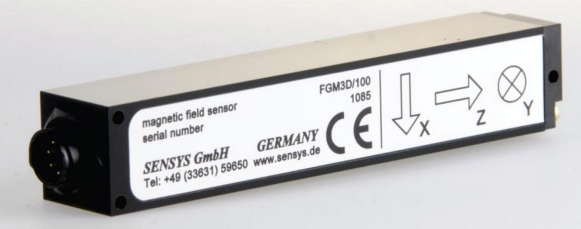

Figure 1. FGM3D fluxgate magnetometer sensor.

\subsection{Fluxgate FGM3D/100 sensor}

A fluxgate magnetic sensor is the main component of this magnetometer. Choosing a lightweight sensor with sufficient accuracy that is low powered and less prone to error is a key point in this consideration. The FGM3D sensor from SENSYS GmbH (Fig. 1) has several advantages, including its waterproof (IP65 certification) and all-weather measurement condition. FGM3D is a three-axis sensor with a core ring type, equipped with a feedback system. Receiving magnetic field as its input, this sensor's output is a three-channel analog signal. Each channel represents a vector component from magnetic field vector input. This transfer function has a sensitivity as small as $0.1 \mathrm{mV} \mathrm{nT}^{-1}$ with a range from -10 to $10 \mathrm{~V}$.

\subsection{Microcontroller}

The microcontroller is the main processor and interconnector of the whole system. All hardware is assembled and connected to this microcontroller. Software which manages acquisition is also installed in this microcontroller. The Arduino platform is one of the most popular and easy-to-use programmable microcontrollers. Utilizing a programmable microcontroller ensures easy reproducibility. Arduino Mega 2560 R3 is used here; it is a development board with the ATmega2560 microcontroller as its core. It is equipped with 54 digital I/O pin, $16 \mathrm{ADC}$, 4 serial gates, $16 \mathrm{MHz}$ crystal oscillator, USB gate, a power jack and reset button. Therefore it has enough port to connect with other devices. ATmega2560 has 8-bit architecture and is capable of working up to 16 million executions in $1 \mathrm{~s}$.

\subsection{Analog-to-digital converter}

The output signal from fluxgate sensor is in the form of an analog signal. To be recorded, processed and digitally displayed, an ADC is required. The output signal from sensor FGM3D is three differential analog signals; therefore an ADC that has at least three differential channels is needed. This key factor requires an ADC with sufficient bit resolution and differential channel support to be paired with FGM3D. The 24-bit ADC ADS1256 from Texas Instruments was chosen for its high bit resolution, differential channel support

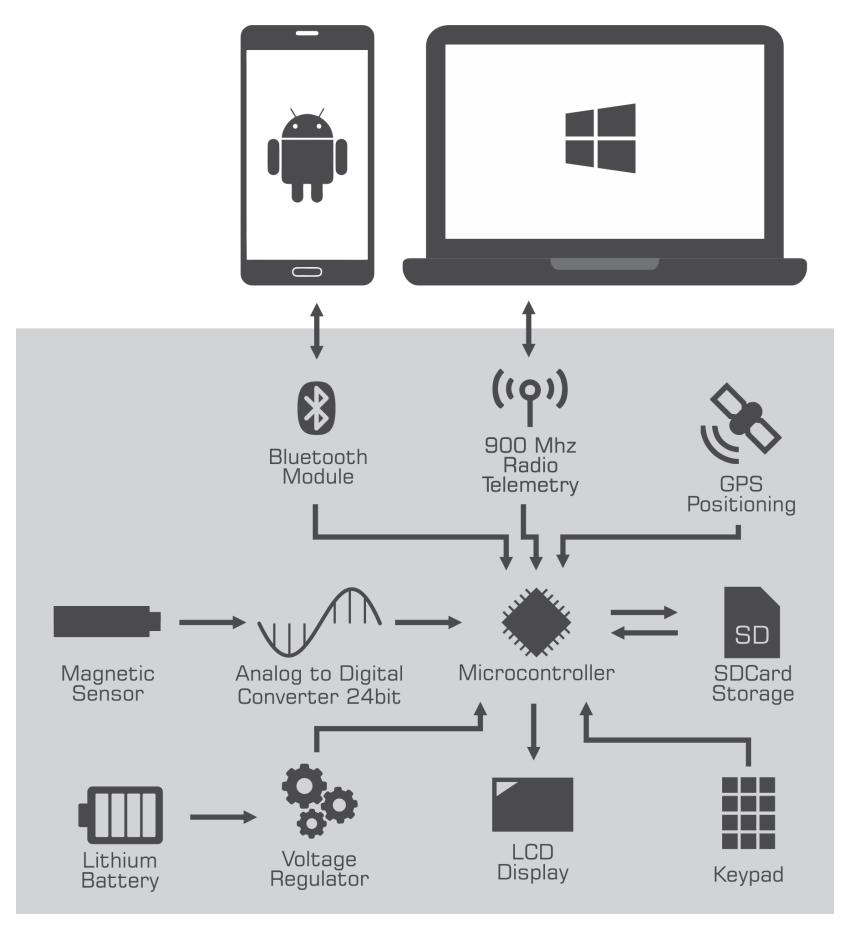

Figure 2. Magnetogama's block diagram.

and very low internal noise. Based on the manufacturer's data sheet, there are 18 bits out of a possible 24-bit output from this ADC that are cleared of internal noise.

\subsection{Data storage and positioning}

Data storage is a fundamental element of an acquisition system that stores output data from an ADC to a nonvolatile memory; no data loss will occur when the device is turned off. Getting and storing location data of the measured point coordinate is also an important aspect. ITEAD GPS shield has both capabilities regarding storage and positioning system. It has one micro-SD slot and a GPS receiver. Digital data from ADC and GPS are written by the shield, which continuously parses GPS data and communicates with the microcontroller through a serial connection into the memory card.

\subsection{Power}

A compatible battery was chosen based on power consumption, ensuring the time needed for acquisition. Another consideration is weight. The battery is heavier than any other part; luckily its capacity is usually proportional to its weight. Due to the plan to mount this acquisition system to a UAV, the battery needed to be as light as possible. A lithium polymer battery was chosen for this device. The whole set of parts requires two battery inputs; the first supplies the FGM3D sensor and the second supplies the microcontroller and any parts connected directly to the microcontroller. 


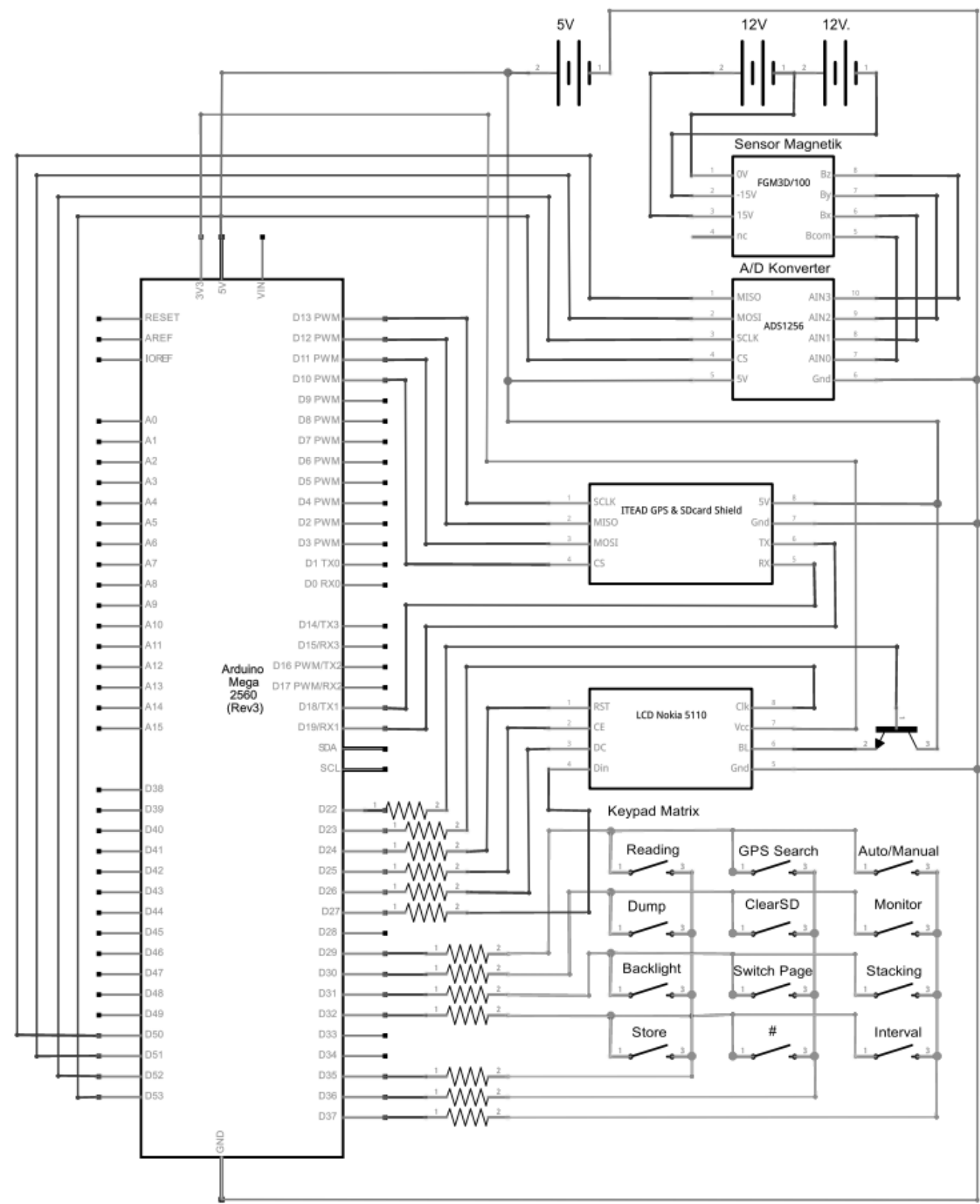

Figure 3. Magnetogama's schematic.

The first power supply consists of two $4 \mathrm{C} 14.8 \mathrm{~V}$ lithium polymer batteries in a serial configuration to supply the positive and negative voltage required by FGM3D. Each battery has a $1300 \mathrm{mAh}$ capacity. The first power supply is connected to LM7812 and LM7912 as a voltage regulator to stabilize input power at $\pm 12 \mathrm{~V}$. The second power supply uses a bigger capacity of $2200 \mathrm{mAh}$, a $7.4 \mathrm{~V} \mathrm{2C}$ lithium polymer battery to power microcontroller along with ADC, GPS and storage shield. No external voltage regulator is required for the second power supply since the internal $5 \mathrm{~V}$ voltage regulator in Arduino Mega 2560 can be used for this purposes.

\subsection{Display and input}

The display on Magnetogama is used to communicate between device and user. Previously, a simple dot matrix display which could only display numeric/character information was sufficient, but it was not intuitive enough for the user. LCD Nokia 5110 is now used for its simplicity and its ability to communicate with the user with more complicated graphics or text. It has a $84 \times 48$ pixel resolution and utilizes the PCD8544 controller, which was also used on the previous Nokia 3310 LCD. This LCD is easy to use and suitable 


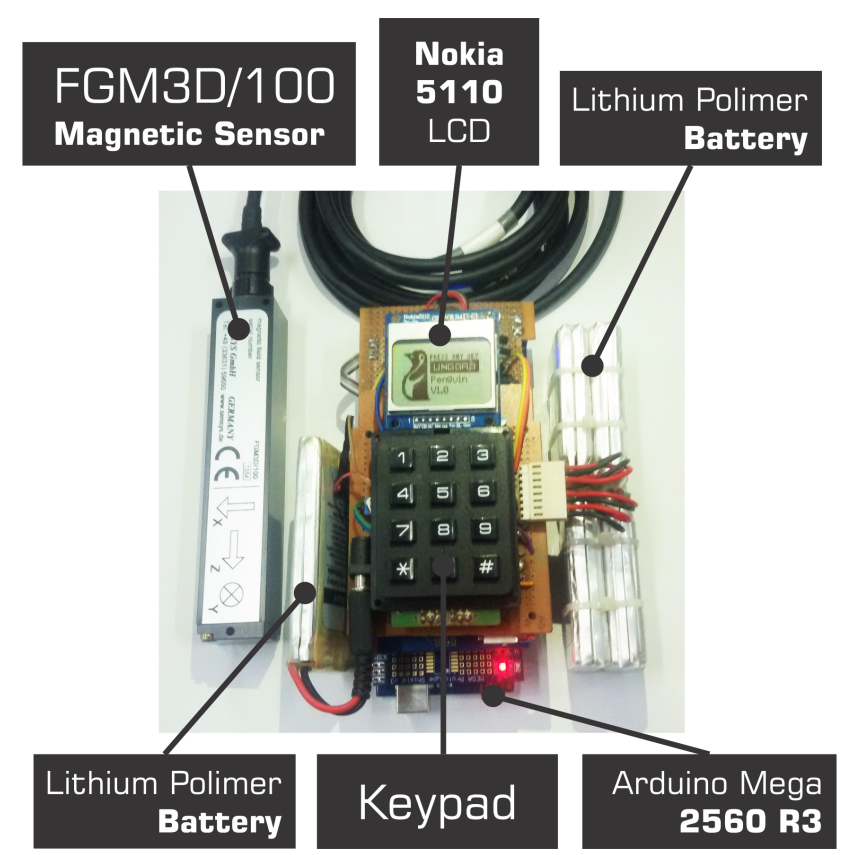

Figure 4. Assembled acquisition tools consist of magnetic sensor FGM3D/100, Arduino Mega 2560 R3, keypad, lithium polymer battery and Nokia 5110 LCD.

for a simple project, as presented by Wagh et al. (2014). User input is provided using a $3 \times 4$ membrane keypad.

\section{Schematic and assembly}

The microcontroller, magnetic sensor and all other parts are assembled following a block diagram and electronic schematic given in Figs. 2 and 3, respectively. The assembled Magnetogama can be seen in Fig. 4. It is important to note that an additional external case is needed to protect the device and perform magnetic survey easily. The case was made from ABS plastic and acrylic as shown in Fig. 5. With the included acrylic case, Magnetogama has a weight of $1 \mathrm{~kg}$ and a dimension of $116 \times 200 \times 65 \mathrm{~mm}$. With given assembly setting and battery, it can be used for $10 \mathrm{~h} 20 \mathrm{~min}$ on full acquisition time, which is sufficient for typical 1-day acquisition.

The electronic components inside the case contain material that is prone to magnetic induction. As a consequence, it may affect the sensor reading and cannot be neglected if located at a close distance from the sensor. Hence, the sensor is connected through $3 \mathrm{~m}$ cable from the case to the sensor. It is important to set the sensor and the case apart at maximum distance allowed by the cable. Figure 6 shows the relative influence of the assembled electronic components on the sensor reading at different distances. The data points are fitted to an exponential equation shown by a solid line. The magnetic signature of electronic component starts to have significant

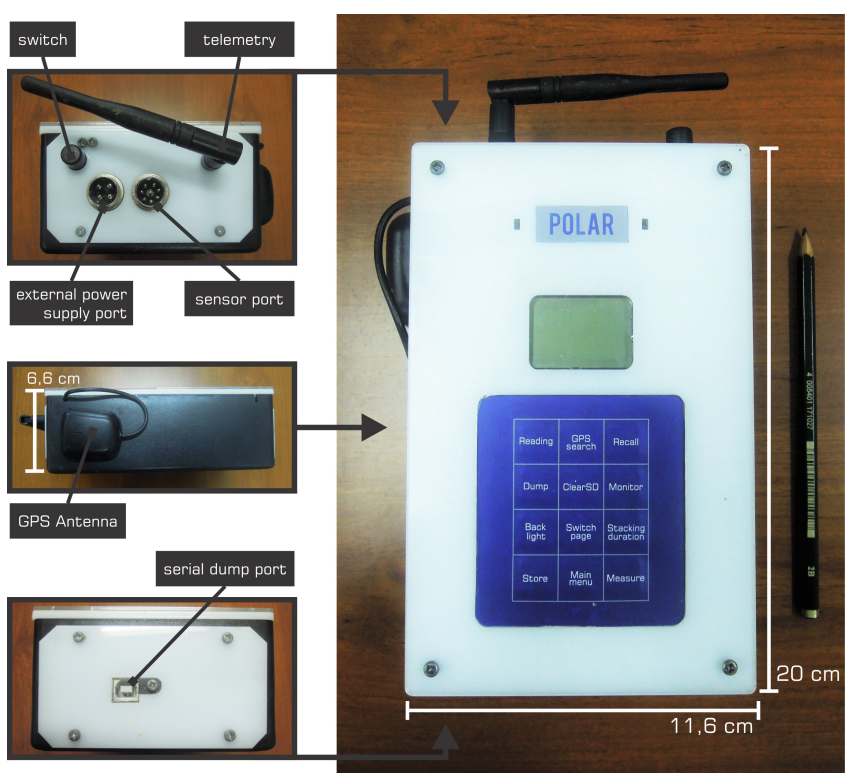

Figure 5. Magnetogama assembled using external plastic case and acrylic cover.

deviation at $260 \mathrm{~cm}$. Therefore, the magnetic reading should not be affected at a minimum distance of $260 \mathrm{~cm}$.

\section{Remote control}

Magnetogama can be controlled locally via its membrane keypad or remotely using wireless serial interface. The wireless interface is switchable between Bluetooth and radio. An Android-based device is used to control Magnetogama via Bluetooth interface. For radio interface, a $915 \mathrm{MHz}$ radio receiver and transmitter is used to control Magnetogama via special PC software developed in this research.

\section{Verification}

\subsection{Comparison with standard magnetometer}

The first step of calibration was performed by comparing continuous measurement recorded with Magnetogama with continuous data from standard magnetometer near the location of the test. Two standard magnetometer observatories located at Learmonth (LRM) and Kakadu (KDU), Australia, were selected as a comparison. The test were performed from UTC 12:00 on 8 February 2016 to UTC 12:00 on 12 February 2016. The test used $0.2 \mathrm{~Hz}$ sampling frequency resulting in 691200 points recorded for each component. The FGM3D sensor was attached to a nonmagnetic tripod using acrylic mounting. The sensor orientation was then set up so the $x$ axis pointed northward, $y$ axis eastward and $z$ axis downward. 
Relative magnetic field influence of magnetogama electronic components

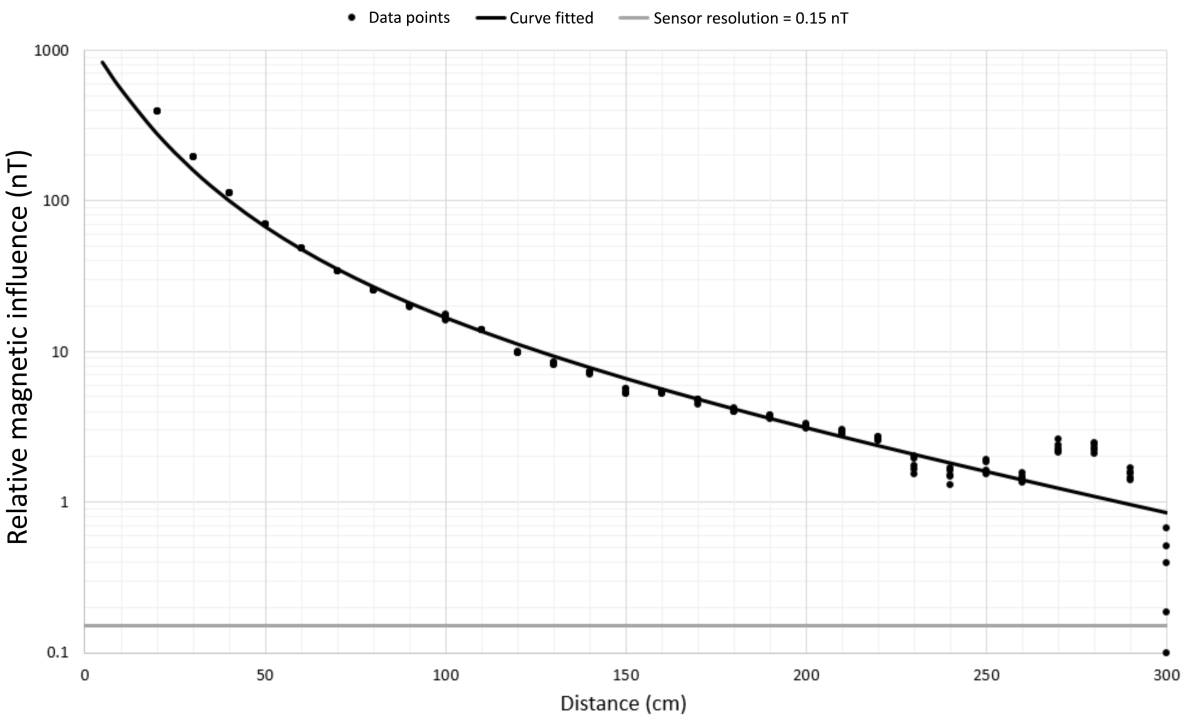

Figure 6. Relative magnetic field influence of Magnetogama to sensor reading over various distances.
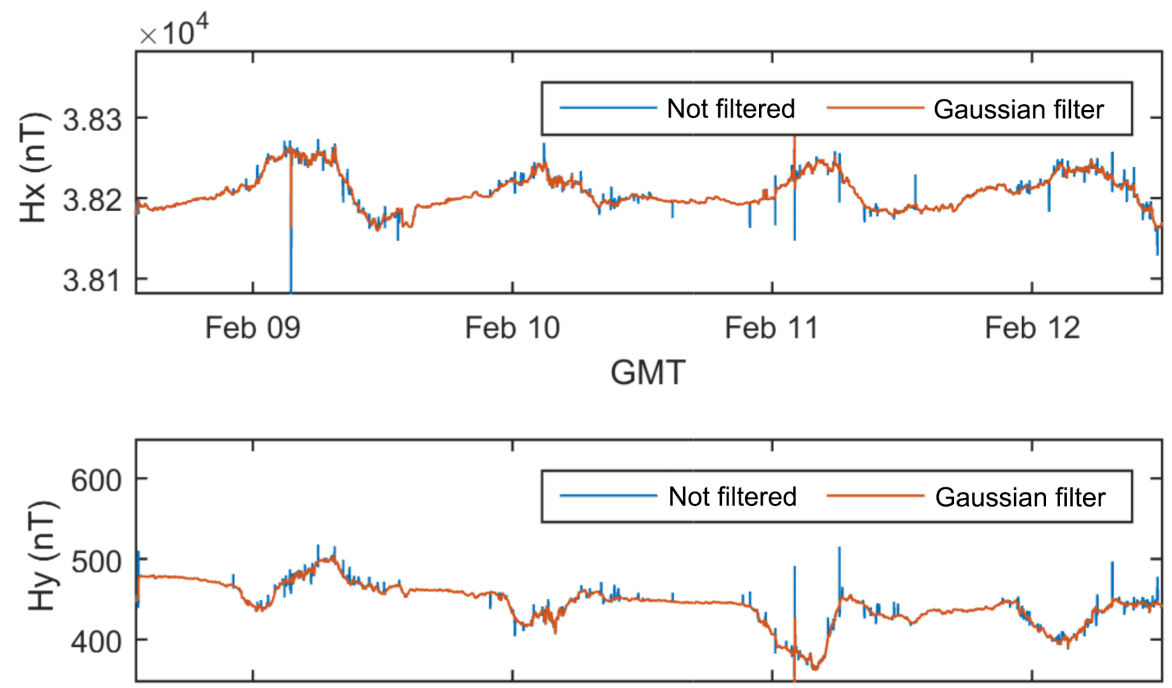

Feb 09

Feb 10

Feb 11

Feb 12

GMT

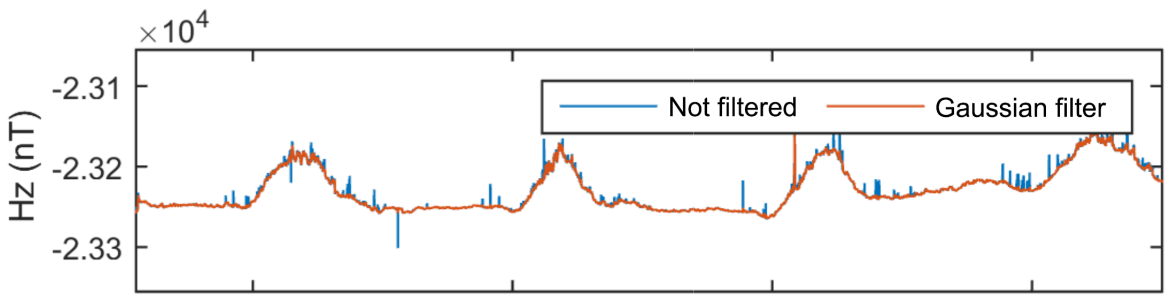

Feb 09

Feb 10

Feb 11

Feb 12

GMT

Figure 7. Comparison between original and filtered Magnetogama (YOG) magnetic field record on each component. 

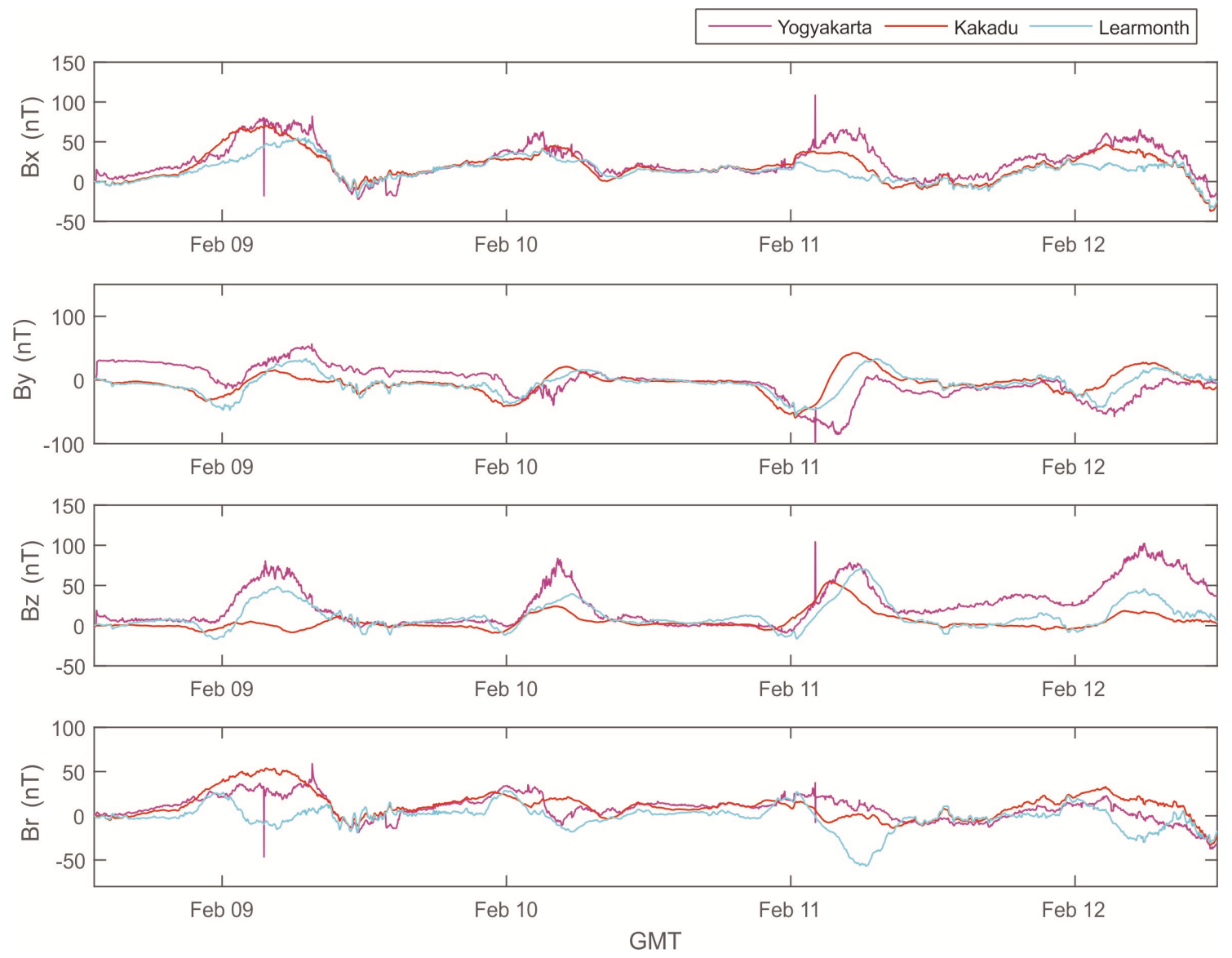

Figure 8. Comparison between Magnetogama (YOG) and magnetometer on Learmonth (LRM) and Kakadu (KDU) observatory.

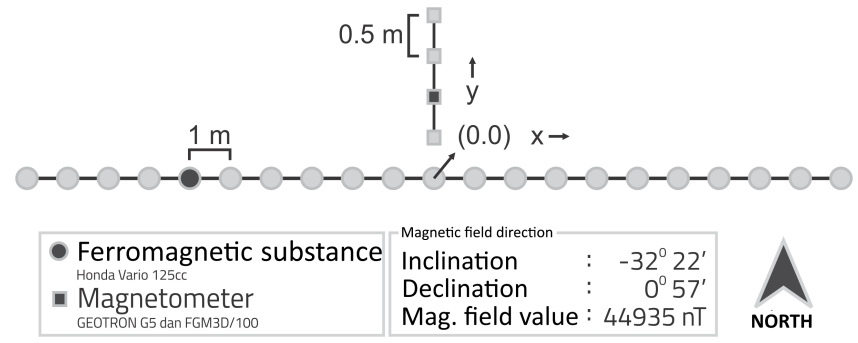

Figure 9. Sketch on Magnetogama's test toward magnetic substance.

Before direct comparison, noise reduction of the tested Magnetogama was carried out. Noise reduction refers to the technical reference manual of the International Geomagnetic Observatory Organization (INTERMAGNET). It is achieved by applying a series of coefficients of a Gaussian filter to the data samples listed in the manual. This digital filter was applied to both standard magnetometers. Figure 7 shows a comparison between original and filtered Magnetogama magnetic field data record.
Figure 8 shows a direct comparison between data recorded on Magnetogama (YOG) and two other reference stations (LRM and KDU). Due to a longitude difference that causes the difference in time and relative sun position, crosscorrelation analysis was performed to estimate diurnal variation pattern. This cross-correlation analysis was meant to determine the signal delay between two different stations as the effect of diurnal variation due to the sun's relative position. Based on cross-correlation analysis, YOG-LRM has a $41 \%$ correlation coefficient and YOG-KDU has $72 \%$. This result is sufficient considering the huge separation distance between those two stations. Therefore, the local disturbance has the main effect on the recorded result.

\subsection{Response of magnetic substance}

Response test of magnetic substance was performed by comparing Magnetogama with a standard magnetometer. In this case, PPM from Geotron G5 was used as a comparison. Magnetometers were located at $x=0 \mathrm{~m}$, with some variation in the $y$ direction $(0.5,1,1.5$ and $2 \mathrm{~m}$ ). A magnetic object (a motor scooter) was initially located at $x=-10 \mathrm{~m}$ and then moved $1 \mathrm{~m}$ for each measurement in the $x$ direction until 

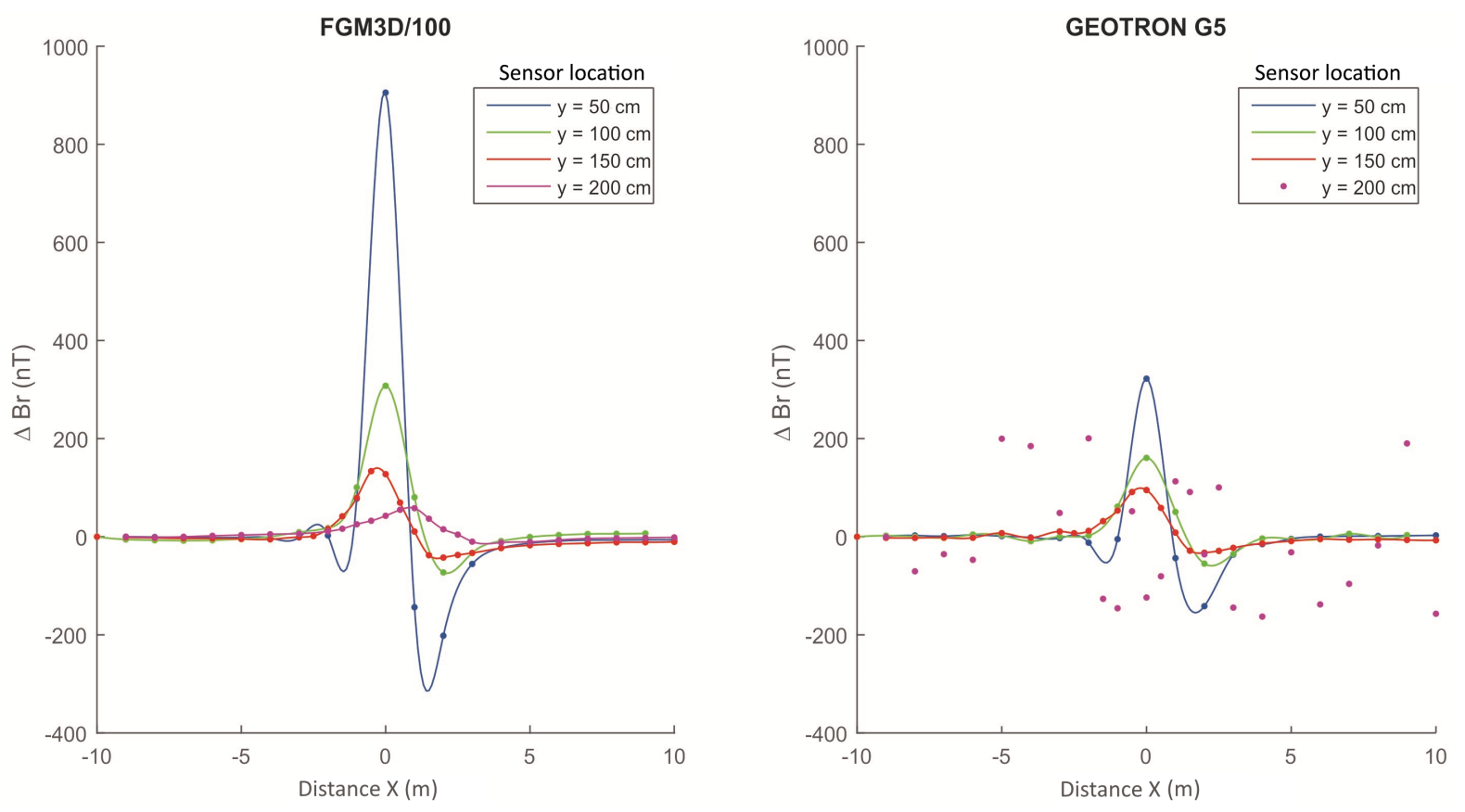

Figure 10. Magnetic field response comparison between FGM3D/100 and PPM Geotron G5.

reaching $x=10 \mathrm{~m}$ (see Fig. 9 for detailed illustration). The magnetization level due to a magnetic object is inversely proportional to the square of its distance and reaches its maximum when a magnetic substance was located at $x=0 \mathrm{~m}$ (Fig. 10).

The amplitude of the graphs in Fig. 10 present the sensor's sensitivity. FGM3D/100 turns out to be more sensitive than Geotron G5, reflected by the higher amplitude on its graph. The variation of the magnetometer's position gives us a stable result with a peak response when a magnetic substance is located at $x=0 \mathrm{~m}$. This result also gives insight into the minimum distance threshold at which an unwanted magnetic object will affect the reading during a real magnetic survey. A partially magnetic object as a big as motor scooter does not show any obvious effect after $5 \mathrm{~m}$ distance. Unfortunately, vector calibration cannot be performed because PPM Geotron G5 is a scalar magnetometer, whereas fluxgate FGM3D/100 is a vector magnetometer. Therefore, for more precise utilization, calibration using another calibrated fluxgate magnetometer is needed.

\section{Conclusions}

Magnetogama is a hand-assembled fluxgate magnetometer utilizing a factory-built-in fluxgate FGM3D/100 sensor combined with Arduino Mega 2650 R3 microcontroller, ADC ADS1256 24-bit, data storage and GPS receiver shield. It is an open schematic and easy to build even with the minimum budget. It has sufficient accuracy to be used for nearsurface geophysics problems, such as mineral exploration and geotechnical issues. Additionally, Magnetogama is designed to be as lightweight as possible $(1 \mathrm{~kg})$ and can be carried by a small UAV.

Code availability. The pseudo-code below represents the general idea of the program inside the microcontroller. Our source code for the microcontroller and ADC can be accessed at https://github.com/ adienakhmad/Magnetogama.

Data availability. Data used in this paper can be accessed at https: //github.com/adienakhmad/Magnetogama. 


\section{Appendix A: Algorithm}

\section{A1 Microcontroller}

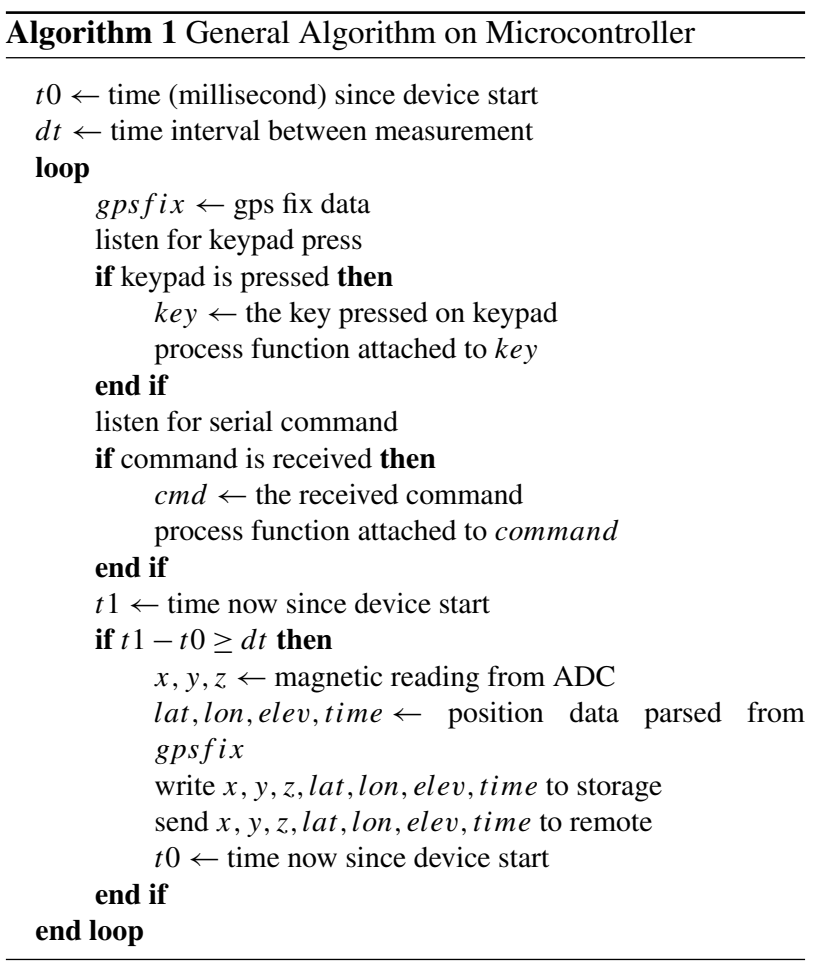

\section{A2 Analog-to-digital converter}

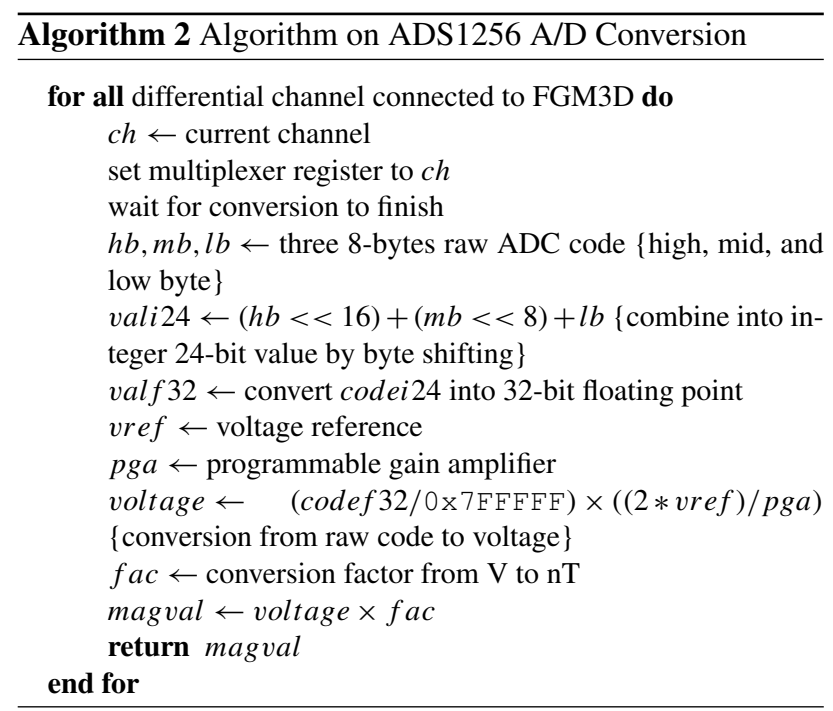


Author contributions. W, NK and TK are research project leader who provide idea and guidance, including the quality check on our apparatus. AR, ASH and AAM designed and test Magnetogama. TMI and all the others help writing the manuscript.

Competing interests. The authors declare that they have no conflict of interest.

Acknowledgements. Authors thank Geophysics Laboratory, Faculty of Geography and Electronics and Instrumentation Laboratory of Universitas Gadjah Mada for the support, http://www.dikti.go.id/ Ministry of Research, Technology, and Higher Education Indonesia for their research fund support, contract no. 781/UN1-P.III/LT.DITLIT/2016. Magnetogama is an open schematic apparatus; therefore the copyright of the product belongs to the authors.

Edited by: Marina Díaz-Michelena

Reviewed by: two anonymous referees

\section{References}

Cleuziou, J.-P., Wernsdorfer, W., Bouchiat, V., Ondarçuhu, T., and Monthioux, M.: Carbon nanotube superconducting quantum interference device, Nat. Nanotechnol., 1, 53-59, 2006.

Drung, D., Cantor, R., Peters, M., Scheer, H., and Koch, H.: Lownoise high-speed dc superconducting quantum interference device magnetometer with simplified feedback electronics, Appl. Phys. Lett., 57, 406-408, 1990.

Fagaly, R.: Superconducting quantum interference device instruments and applications, Rev. Sci. Instrum., 77, 101101-1101101-45, 2006.

Peeters, F. and Li, X.: Hall magnetometer in the ballistic regime, Appl. Phys. Lett., 72, 572-574, 1998.

Primdahl, F.: The fluxgate magnetometer, J. Phys. E-Sci. Instrum., 12, 241-253, https://doi.org/10.1088/0022-3735/12/4/001, 1979.

Serson, P.: Proton precession magnetometer, uS Patent 3,070,745, 1962.

Tyagi, S., Lord, A., and Koerner, R.: Use of a proton precession magnetometer to detect buried drums in sandy soil, J. Hazard. Mater., 8, 11-23, 1983.

Wagh, A., Dave, Z., Singh, G., Dange, V., Tambe, A., and Gengaje, S.: A low cost portable oscilloscope for educational platforms using a Programmable System on Chip, in: Advances in Communication and Computing Technologies (ICACACT), 2014 International Conference on, 1-4, IEEE, 2014. 